\title{
Uso de agua de rechazo de plantas depuradoras en la fabricación de hormigones
}

\author{
Use of waste water from purification plants in the manufacture of concrete
}

Fecha de entrega: 6 de agosto 2018

Fecha de aceptación: 31 de octubre 2018

\section{Amin Nazer ${ }^{1,3}$, Alejandro Guzmán ${ }^{1}$, Luis Bolados ${ }^{1}$, Luis González ${ }^{1}$ y Osvaldo Pavez ${ }^{2,3}$}

\author{
${ }^{1}$ Departamento de Construcción, Facultad Tecnológica, Universidad de Atacama, Avenida Copayapu 485, Copiapó, Chile, \\ amin.nazer@uda.cl, alejandro.guzman@uda.cl, luis.bolados@uda.cl, luis.gonzalez@uda.cl \\ ${ }^{2}$ Departamento de Ingeniería en Metalurgia, Facultad de Ingeniería, Universidad de Atacama, Avenida Copayapu 485, Copiapó, Chile, \\ osvaldo.pavez@uda.cl \\ ${ }^{3}$ CRIDESAT, Universidad de Atacama, Avenida Copayapu 485, Copiapó, Chile,
}

La Región de Atacama presenta ciclos de escasez hídrica que afectan tanto a la población como a los sectores productivos, los que demandan ingentes cantidades de agua potable para su desarrollo. Con el propósito de disminuir la presión sobre el recurso hídrico, se incentiva al ahorro del agua potable y/o la reutilización de aguas grises domiciliarias y aguas industriales. En este contexto, la utilización de agua de rechazo proveniente de los procesos de purificación de agua salobre, se presenta como una buena alternativa al agua potable en la fabricación de morteros y hormigones. El presente estudio evalúa el comportamiento mecánico del hormigón conteniendo agua de rechazo generada en los procesos de depuración por osmosis inversa e intercambio iónico. Para ello, se caracterizaron 4 tipos de agua: agua potable (control), agua de rechazo del proceso de osmosis inversa utilizado en la producción de agua purificada para la bebida; agua de rechazo tanto del proceso de intercambio iónico como del proceso de osmosis inversa, ambos del Hospital Regional de Copiapó, Atacama, Chile. Posteriormente, se fabricaron diferentes muestras de hormigón conteniendo los 4 tipos de agua y se evaluó su resistencia a la compresión a las edades de 7 y 28 días. Los resultados mostraron que, en todos los casos, los hormigones fabricados con agua de rechazo alcanzaron mayores resistencias a la compresión que la muestra control. De los resultados del estudio, se puede concluir que el uso de agua de rechazo en la fabricación de hormigones, puede ser una conveniente alternativa para la construcción en zonas áridas.

Palabras clave: osmosis inversa, intercambio iónico, hormigón, agua de rechazo
The Atacama Region presents cycles of water scarcity that affect both the population and the productive sectors, which demand huge amounts of drinking water for its development. In order to reduce the pressure on the water resource, it encourages the saving of drinking water and/or the reuse of sewage and industrial waters. In this context, the use of waste water from the brackish water purification processes is a good alternative to drinking water in the manufacture of mortars and concretes. The present study evaluates the mechanical behaviour of the concrete containing waste water generated in the processes of reverse osmosis and ion exchange. To do this, four types of water were characterized: drinking water (control), reverse osmosis waste water process used in the production of purified drinking water; waste water of both the ion exchange process and the reverse osmosis process, both from the Regional Hospital of Copiapó, Atacama, Chile. Subsequently, different samples of concrete containing the 4 types of water were manufactured and their resistance to compression at the ages of 7 and 28 days was evaluated. The results showed that in all cases, the concrete made with waste water, reached higher resistance to compression than the control sample. From this study, it can be concluded that the use of waste water in the manufacture of concrete, can be a convenient alternative for the construction in arid zones.

Keywords: reverse osmosis, ion exchange, concrete, waste water

\section{Introducción}

La Región de Atacama se encuentra en una zona árida del norte de Chile, lugar en donde la escasez hídrica se ha incrementado notoriamente en los últimos años. Es por 
ello que resulta muy conveniente incentivar el ahorro del agua y/o la reutilización de aguas residuales de la industria en otros procesos. En este contexto, la utilización de agua de rechazo proveniente de procesos de purificación de agua salobre, sea mediante destilación térmica, congelación, ósmosis inversa o intercambio iónico, toma importancia y puede considerarse como una nueva fuente hídrica no potable. En el proceso de purificación de agua salobre se obtienen dos fases acuosas: una fase la constituye el agua dulce conteniendo bajos niveles de sales, y otra fase salina o salmuera que es eliminada, generalmente sin darle uso. El vertido de la salmuera ocasiona impactos ambientales al suelo y al agua marina afectando a la vida vegetal y animal del entorno.

Un posible uso del agua residual puede ser en el sector de la construcción, particularmente como un componente en la fabricación de elementos de hormigón. El hormigón es una mezcla de grava, arena, cemento y agua potable, dosificadas convenientemente para lograr materiales resistentes a esfuerzos, durables e impermeables, entre otras cualidades. El agua en el hormigón, participa activamente en la reacción química con el cemento formando un conglomerado que endurece en el tiempo. El agua utilizada en morteros y hormigones, generalmente es agua potable, la cual regula la trabajabilidad, la microestructura, la resistencia, la durabilidad general del hormigón, favoreciendo, además, la hidratación del cemento.

En la literatura se reportan investigaciones que evalúan el uso de diferentes tipos de agua en la fabricación de materiales de construcción: agua potable, agua magnetizada (Bharath et al., 2016; Guimarães, 2006), aguas residuales (Ramkar y Ansari, 2016; Al-Jabri et al., 2011) y agua de alcantarillado (Nikhil et al., 2014). Se prefiere utilizar el agua potable por no contener impurezas que pudieran afectar la calidad del hormigón. Al respecto, Kucche et al. (2015) indican que las impurezas presentes en el agua reaccionan de manera diferente con los componentes del cemento, pudiendo afectar principalmente el tiempo de fraguado y la resistencia a la compresión.

Por otra parte, la presencia de sales de sulfatos presentes en el agua residual, puede afectar al hormigón modificando sus características de diseño, causando expansiones y fisuraciones al formarse monosulfoaluminato y etringita por el sulfato sódico $\mathrm{NaSO}_{4}$ e hidróxido de magnesio (brucita), y sulfato de calcio $\mathrm{CaSO}_{4}$ (yeso), debido a la presencia del sulfato magnésico $\mathrm{MgSO}_{4}$ (Bernal et al., 2013). Además, los sólidos totales disueltos reducen la resistencia y durabilidad del material de construcción (Halder et al., 2015).

En el presente estudio, se evaluó el comportamiento mecánico a la compresión de muestras de hormigón fabricadas con sustitución del 0\% (control) y 100\% de agua potable por agua de rechazo generada en 3 diferentes procesos de purificación de agua salina proveniente de equipos de ósmosis inversa e intercambio iónico.

\section{Materiales}

Para los ensayos a la compresión se fabricaron probetas cúbicas de $15 \mathrm{~cm}$ de arista con resistencias características de hormigones $\mathrm{H}_{10}(10 \mathrm{MPa})$ y $\mathrm{H}_{20}(20 \mathrm{MPa})$.

\section{Cemento y áridos}

En la fabricación de las probetas de hormigón, se utilizó cemento puzolánico Inacesa Especial, y tres tipos de áridos: grava con tamaño máximo $40 \mathrm{~mm}$, gravilla y arena.

La distribución granulométrica de los áridos cumple con la NCh163 (1979). La distribución granulométrica de los áridos empleados en la fabricación de los hormigones se muestra en la Figura 1.

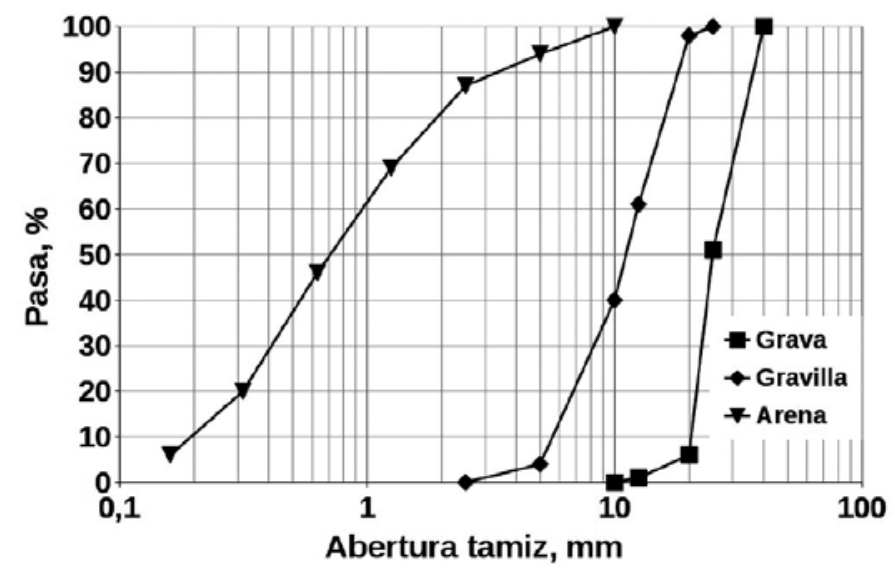

Figura 1: Distribución granulométrica de los áridos utilizados en hormigones

En la Tabla 1 se muestra la dosificación de los materiales utilizados en el diseño de hormigones $\mathrm{H}_{10} \mathrm{y} \mathrm{H}_{20}$. 
Tabla 1: Dosificación de materiales para $1 \mathrm{~m}^{3}$ de hormigón

\begin{tabular}{|c|c|c|}
\hline Materiales & $\begin{array}{c}\mathrm{H}_{10} \\
\text { litros }\end{array}$ & $\begin{array}{c}\mathrm{H}_{20} \\
\text { litros }\end{array}$ \\
\hline Agua & 170 & 170 \\
\hline Cemento & 81 & 103 \\
\hline Grava & 296 & 287 \\
\hline Gravilla & 185 & 179 \\
\hline Arena & 258 & 251 \\
\hline
\end{tabular}

\section{Agua}

En este estudio se utilizaron los siguientes tipos de agua: agua potable de la ciudad de Copiapó (Agua 1), agua de descarte de un proceso de ósmosis inversa aplicado en la producción de agua purificada (Agua 2), agua de descarte de un proceso de intercambio iónico utilizado en el Hospital Regional (Agua 3); y Agua de descarte de un proceso de ósmosis inversa utilizado en el laboratorio del Hospital Regional (Agua 4).

\section{Equipos utilizados en la producción de agua purificada}

En el proceso de producción de agua purificada en el laboratorio del Hospital Regional, se utilizó un equipo de ósmosis inversa modelo CILIT y un equipo de intercambio iónico modelo CILIT Super Data/Biodata. Además, se utilizó un equipo de ósmosis inversa General Electric E4413200-DLX, de una empresa productora de agua purificada para la bebida.

\section{Métodos}

\section{Caracterización química del agua}

En los diferentes tipos de agua utilizadas en este estudio, se analizó lo siguiente: $\mathrm{pH}$, sólidos disueltos totales (SDT), conductividad eléctrica CE, dureza, temperatura, calcio, carbonato, cloruro, magnesio, sulfatos. Todas las mediciones fueron realizadas en el laboratorio de la empresa QUIMAT.

\section{Fabricación de probetas y ensayos de resistencia a la compresión}

Una vez planificadas las actividades y teniendo a disposición todos los materiales $\mathrm{y}$ herramientas necesarias, se procedió a fabricar los hormigones de prueba siguiendo los procedimientos indicados en la NCh1018 (1977). El hormigón de prueba se preparó mediante mezclado mecánico, utilizando una mezcladora de 90 litros de capacidad. Respecto de los ensayos de resistencia a la compresión, éstos se realizaron de acuerdo al procedimiento señalado en la NCh1037 (1977). Todos los ensayos fueron realizados en el Laboratorio Control y Ensayes de Materiales S.A. (LACEM S.A.), Copiapó.

\section{Resultados}

En la Tabla 2 se muestran los resultados de los análisis realizados a los 4 tipos de agua. Se aprecia que el agua de rechazo presenta valores superiores al Agua 1, en los siguientes análisis: dureza, calcio, cloruro y magnesio. Además, los resultados de conductividad eléctrica y sólidos disueltos totales SDT para el Agua 1 fueron inferiores que en las muestras de agua de rechazo, a excepción del Agua 2; mientras que los valores de sulfatos fueron menores en el Agua 3. La presencia de carbonatos fue menor a 0.02 $\mathrm{mg} / \mathrm{L}, \mathrm{y}$ los valores $\mathrm{pH}$ y temperatura fueron bastante similares.

Por otra parte, todos los tipos de agua de rechazo estudiadas cumplen con los requisitos para el agua de amasado para hormigones establecido en la norma chilena NCh1498 (1982).

Tabla 2: Propiedades del agua utilizada en hormigones

\begin{tabular}{|l|c|c|c|c|}
\hline Propiedades & Agua 1 & Agua 2 & Agua 3 & Agua 4 \\
\hline $\mathrm{pH}$ & 7.54 & 7.64 & 6.89 & 7.49 \\
\hline $\mathrm{CE}, \mu \mathrm{S} / \mathrm{cm}$ & 2896 & 2550 & 3247 & 3402 \\
\hline $\mathrm{SDT}, \mathrm{mg} / \mathrm{L}$ & 1882 & 1641 & 2110 & 2211 \\
\hline Dureza, $\mathrm{mg} / \mathrm{L}$ & 1170 & 2510 & 1230 & 1390 \\
\hline Calcio, mg/L & 299.0 & 539.0 & 316.0 & 322.6 \\
\hline Cloruro, $\mathrm{mg} / \mathrm{L}$ & 304.8 & 606.9 & 400.8 & 324.0 \\
\hline Magnesio, $\mathrm{mg} / \mathrm{L}$ & 83.4 & 145.0 & 87.8 & 87.9 \\
\hline Sulfato, $\mathrm{mg} / \mathrm{L}$ & 940.2 & 1622.5 & 934.1 & 977.0 \\
\hline Carbonato, $\mathrm{mg} / \mathrm{L}$ & $<0.02$ & $<0.02$ & $<0.02$ & $<0.02$ \\
\hline Temperatura $^{\circ} \mathrm{C}$ & 23.6 & 23.4 & 23.5 & 23.4 \\
\hline
\end{tabular}

En las Figuras 2 y 3 se muestran las resistencias a la compresión de hormigones $\mathrm{H}_{10} \mathrm{y} \mathrm{H}_{20}$, respectivamente, a las edades de 7 y 28 días, para los diferentes tipos de agua utilizadas. Se observa en ambas figuras, que los mejores resultados son obtenidos con el Agua 3 y el Agua 4.

En las Figuras 2 y 3 se aprecia que los valores de resistencia a la compresión a los 7 y 28 días, obtenidos en los hormigones conteniendo Agua 1 y Agua 2, son 
bastante menores que los alcanzados con los hormigones conteniendo Agua 3 y Agua 4.

También debe señalarse que, para los diferentes tipos de agua utilizadas en los ensayos, todos los valores de resistencia a la compresión obtenidos en los ensayos de hormigón son mayores que el control.

Observando en la Figura 2 los resultados de resistencia a la compresión de los hormigones $\mathrm{H}_{10}$, conteniendo Agua $3 \mathrm{y}$ Agua 4, éstos muestran incrementos respecto del hormigón fabricado con Agua 1, de $170 \%$ y $212 \%$ a los 7 días y $71 \%$ y $79 \%$ a los 28 días, respectivamente. De igual forma, en la Figura 3, se observan incrementos del hormigón $\mathrm{H}_{20}$ respecto al hormigón control, de $74 \%$ y $91 \%$ a los 7 días y $43 \%$ y $51 \%$ a los 28 días en hormigones conteniendo Agua 3 y Agua 4, respectivamente.

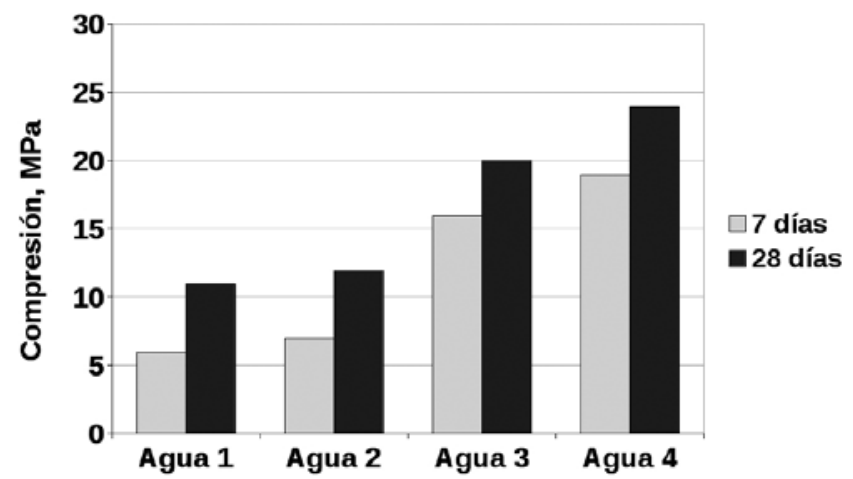

Figura 2: Resistencia a la compresión en función de las edades (7 y 28 días), para hormigones $\mathrm{H}_{10}$

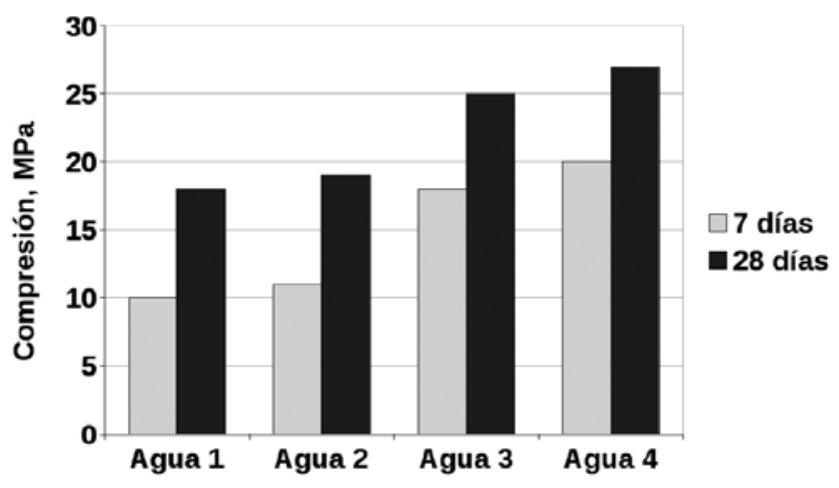

Figura 3: Resistencia a la compresión en función de las edades (7 y 28 días), para hormigones $\mathrm{H}_{20}$

Respecto a las resistencias a la compresión de hormigones $\mathrm{H}_{10}$ y $\mathrm{H}_{20}$ conteniendo Agua 2, se observaron resultados inferiores a los hormigones con Agua 3 y Agua 4 a edades de 7 y 28 días.

Esto podría explicarse por los altos contenidos de sulfatos, cloruros y magnesio contenidos en el Agua 2 (Tabla 2), causantes de la formación de etringita y sulfatos de calcio y magnesio que causarían la disminución de la resistencia a la compresión.

\section{Discusión}

Reciclar y reutilizar aguas residuales es importante para evitar problemas ambientales y el desperdicio de este recurso hídrico. Además, en varias investigaciones se ha encontrado que reutilizar aguas residuales en la mezcla de hormigón es una alternativa posible (e.g. Sabriani, 2010). Kucche et al. (2015) indican que hay varias fuentes de agua, existentes y nuevas, que pueden ser apropiadas para el reemplazo parcial o total de agua potable en la fabricación de hormigón. En estas fuentes se incluyen, aguas recuperadas, aguas subterráneas, aguas tratadas desde aguas negras, aguas de premezclado de hormigón, entre otros. Si bien, las aguas residuales pueden ser utilizadas en mezcla de hormigón, la calidad de este recurso hídrico juega un papel importante en el proceso de preparación del hormigón, ya que los elementos presentes en el agua pueden actuar en las reacciones químicas e interferir en la configuración del cemento y también afectar negativamente la resistencia y la durabilidad del hormigón (Kucche et al., 2015). En estudios realizados por Al-Jabri et al. (2011) se analizó el $\mathrm{pH}$, sólidos disueltos totales, cloruro, dureza, alcalinidad y sulfatos en muestras de aguas residuales provenientes de tres estaciones de lavado de auto, incluyendo también agua potable de grifo. Estos investigadores informaron que, aunque la composición química de las aguas residuales presentaba parámetros de valores superiores a los del agua de grifo, los valores se encontraban dentro de los límites establecidos por AASHTO T26-79 (2008), indicando que las aguas residuales producidas pueden ser usadas satisfactoriamente en mezclas de hormigón. Por otra parte, Sabriani (2010) indica que aguas con exceso de impurezas no solamente puede afectar el tiempo de asentamiento y resistencia del hormigón, sino que también pueden causar eflorescencia, manchas, corrosión, inestabilidad de volumen y menor durabilidad, incluso el uso de agua impura en la preparación de hormigón puede llegar a causar una falla prematura de la estructura. En este contexto, Kucche 
et al. (2015) señala que todas las impurezas puede que no presenten efectos adversos en las propiedades del hormigón y en algunos casos el resultado neto puede ser inofensivo o de mejoras en las propiedades del hormigón. Esto indicaría que es difícil llegar a una conclusión común para el uso de diferentes aguas en mezclas y curado de hormigón. En el presente trabajo, los valores de resistencia a la compresión obtenidos en mezclas de hormigón preparadas con el Agua 3 y Agua 4 fueron muy superiores a los alcanzados con el Agua 1. En ambas aguas de desecho los contenidos de impurezas presentes, así como los valores de CE y SDT, fueron en general superiores a los valores del Agua 1, por lo tanto, la menor calidad del Agua 3 y Agua 4 no significó efectos adversos en las propiedades de las mezclas, sino que resultó en mejoras significativas de la resistencia a la compresión del hormigón. Estos resultados confirmarían lo señalado por Kucche et al. (2015), en el sentido de que resulta difícil concluir sobre el comportamiento que tendrán aguas residuales de menor calidad que el agua potable, en las propiedades de las mezclas de hormigón.

Un parámetro cuyos efectos pueden modificar notoriamente las propiedades de las mezclas es el $\mathrm{pH}$ de las aguas usadas en la preparación del hormigón, por lo cual debe ser cuidadosamente manejado. Kucche et al. (2015) señalan que al disminuir el $\mathrm{pH}$ del agua hay una reducción de la resistencia a la compresión y resistencia a la tracción del hormigón, mientras que con el aumento del $\mathrm{pH}$ del agua se produce una disminución de la fuerza de adherencia y de la acción congelación-descongelación del hormigón reforzado con fibra. Por otra parte, Nikhil et al. (2014) indicaron que, al aumentar el pH del agua, la resistencia en el hormigón disminuye sustancialmente, estos investigadores señalan que debido al valor del $\mathrm{pH}$ de las aguas residuales y la alta presencia de sólidos suspendidos, el silicato de calcio hidratado, que es el responsable de la resistencia en el hormigón, se deteriora y debilita el hormigón. También en relación al efecto del pH en las mezclas de hormigón, Mehta y Monteiro (2013) informaron que cuando la permeabilidad del hormigón es baja y el pH del fluido está por encima de 6, la tasa de ataque químico es bastante lenta. Estos investigadores señalan que el ataque químico sobre el hormigón tiene como resultado efectos físicos perjudiciales, tales como, efectos en la porosidad, disminución de la resistencia, agrietamiento y rompimiento en pedazos. En el presente estudio, la Tabla 2 muestra que las cuatro muestras de agua utilizadas presentaron valores de $\mathrm{pH}$ muy similares (entre 7 y 8), por lo tanto, al no existir una diferencia apreciable en los valores, el efecto del $\mathrm{pH}$ de las aguas utilizadas en la mezcla de hormigón debió ser muy similar.

En los resultados de resistencia a la compresión alcanzados en este estudio, tanto en el hormigón $\mathrm{H}_{10}$ como $\mathrm{H}_{20}$ la utilización de las aguas de rechazo (Agua 3 y Agua 4) permitieron la obtención de mejores resultados que el agua potable, incrementándose claramente la resistencia a la compresión de los hormigones, y en todos los casos los valores obtenidos fueron superiores a los alcanzados en el hormigón control. En la literatura se han informado resultados favorables y también desfavorables sobre el uso de aguas residuales en mezclas de hormigones. Nikhil et al. (2014) informaron sobre diferentes propiedades de una mezcla de hormigón grado M20, utilizando agua potable, agua subterránea y aguas residuales, indicando que el agua potable otorga buenas propiedades de resistencia en el hormigón, observándose un aumento del $33 \%$ en la resistencia a la compresión y $11 \%$ en la resistencia a la flexión, en comparación al hormigón preparado con aguas residuales. Ramkar y Ansari (2016) estudiaron el efecto de diferentes tipos de aguas residuales recogidas de plantas de tratamiento de aguas, con respecto al agua potable en mezclas de hormigón M30. En este estudio el agua potable se reemplazó $100 \%$ por agua residual de un tratamiento primario, agua residual de un tratamiento secundario (STWW) y agua residual doméstica. Estos investigadores informaron que la resistencia a la compresión aumentó al utilizar agua STWW y agua residual doméstica, mientras que en los ensayos de resistencia a la tracción y flexión se obtuvieron los mismos resultados, no observándose mejoras en dichas resistencias. En una investigación realizada por Sabriani (2010), se usó agua de lavado residual en remplazo de agua de grifo y se estudió la resistencia a la compresión del hormigón y sus propiedades. Los resultados de los ensayos realizados reemplazando $100 \%$ el agua del grifo por agua de lavado residual en la mezcla de hormigón, mostraron que el uso del agua de lavado residual mejoraba el rendimiento del hormigón en términos de resistencia a la compresión, absorción de agua y porosidad. Por otra parte, Al-Jabri et al. (2011) prepararon 
mezclas de hormigón usando diferentes porcentajes (25 a $100 \%$ ) de agua residual proveniente de tres estaciones de lavado de auto en remplazo de agua de grifo, concluyendo que la resistencia del hormigón en las mezclas preparadas con aguas residuales fue similar a la resistencia de la mezcla de control. Además, señalaron que la utilización de aguas residuales no afectaba la absorción de agua en el hormigón. Reddy Babu et al. (2009) informaron que mezclas preparadas con aguas residuales de la industria de galvanizado no mostraron pérdida de resistencia, aunque el tiempo de asentamiento aumentó. Estos investigadores señalaron que, en presencia de alta concentración de iones metálicos, la resistencia a la compresión y flexión aumentó levemente. En estudios realizados con morteros, Silva y Naik (2010) indicaron que morteros preparados con aguas residuales de una planta de tratamiento de aguas (efluente de tratamiento secundario) mostraron una mejora en la resistencia a la compresión durante las edades de 3 a 28 días, al compararlo con morteros preparados con agua potable. Estos autores señalan que los resultados sugieren que el contenido orgánico presente en las aguas residuales podría estar actuando como un agente dispersante, mejorando la dispersión de las partículas de cemento y reduciendo la aglutinación.

\section{Conclusiones}

La utilización de agua de rechazo en hormigones simples demostró que es una buena alternativa al agua potable. Esto se verificó con los resultados de resistencia a la compresión de los hormigones, que en todos los casos fueron superiores al del hormigón control. Sin embargo, para su aplicación en el sector de la construcción, se requieren mayores estudios que permitan conocer su durabilidad, especialmente en hormigones armados. Además, el presente estudio, permite sugerir que el uso de agua de rechazo contribuiría a la reducción del consumo de agua potable en materiales de construcción fabricados en zonas áridas.

\section{Referencias}

AASHTO T26-79 (2008). Standard method of test for quality of water to be used in concrete. American Association of State Highway and Transportation Officials. Washington DC, USA
Al-Jabri, K.S., Al-Saidy, A.H., Taha, R. and Al-Kemyani, A.J. (2011). Effect of using wastewater on the properties of high strength concrete. Procedia Engineering 14, 370-376

Bernal, J., Mahmoud, S., Reyes, E. and Moragues, A. (2013). Estudio de la influencia de los medios con presencia de sulfatos en hormigones con cementos sulforresistentes y adiciones minerales. Parte 1: Hormigones expuestos a sulfato sódico $\left(\mathrm{Na}_{2} \mathrm{SO}_{4}\right)$. Revista de la Construcción 12(2), 14-29

Bharath, S., Subraja, S. and Arun Kumar, P. (2016). Influence of magnetized water on concrete by replacing cement partially with copper slag. Journal of Chemical and Pharmaceutical Sciences 9(4), 2791-2795

Guimarães, C.O. (2006). Avaliação do uso da água magnetizada na produção de concretos em centrais. Tesis de mestrado, Universidade Federal de Minas Gerais, Belo Horizonte, Brasil

Halder, B.K., Tandon, V., Chintalapalle, R.V., Roy, D. and Tarquin, A. (2015). A potential biological approach for sustainable disposal of total dissolved solid of brine in civil infrastructure. Construction and Building Materials 76, 51-60

Kucche, K.J., Jamkar, S.S. and Sadgir P. A. (2015). Quality of water for making concrete: a review of literature. International Journal of Scientific and Research Publications 5(1), 1-10

Mehta, P.K and Monteiro, P.J.M. (2013). Concrete: microstructure, properties, and materials. McGraw-Hill

NCh163 (1979). Áridos para morteros y hormigones - Requisitos generales. Instituto Nacional de Normalización INN, Santiago, Chile

NCh1018 (1977). Hormigón - Preparación de mezclas de prueba en laboratorio. Instituto Nacional de Normalización INN, Santiago, Chile

NCh1037 (1977). Hormigón - Ensayo de compresión de probetas cúbicas y cilíndricas. Instituto Nacional de Normalización INN, Santiago, Chile

NCh1498 (1982). Hormigón: agua de amasado: requisitos. Instituto Nacional de Normalización INN, Santiago, Chile

Nikhil, T.R., Sushma, R., Gopinath, S.M. and Shanthappa, B.C. (2014). Impact of water quality on strength properties of concrete. Indian Journal of Applied Research 4(7), 197-199 
Ramkar, A.R. and Ansari, U.S. (2016). Effect of treated waste water on strength of concrete. Journal of Mechanical and Civil Engineering 13(6), 41-45

Reddy Babu, G., Sudarsana Rao, H. and Ramana Reddy, I.V. (2009). Effect of metal ions in industrial wastewater on cement setting, strength development and hardening. Indian Concrete Journal 83, 43-48

Sabriani, S. (2010). The effect of waste wash water on concrete properties. Thesis degree of Bachelor of Civil Engineering, University Malaysia Pahang
Silva, M. and Naik, T.R. (2010). Sustainable use of resources - recycling of sewage treatment plant water in concrete. Proceeding of the Second International Conference on Sustainable Construction Materials and Technologies, J. Zachar, T.R. Naik, E. Ganjian, eds., Universita Politecnica delle Marche, Ancona, Italy 